\title{
The Impaired Bone Anabolic Effect of PTH in the Absence of Endogenous FGF2 is Partially Due to Reduced ATF4 Expression
}

\author{
Yurong Fei \\ University of Connecticut School of Medicine and Dentistry \\ Liping Xiao \\ University of Connecticut School of Medicine and Dentistry \\ Marja M. Hurley \\ University of Connecticut School of Medicine and Dentistry
}

Follow this and additional works at: https://opencommons.uconn.edu/uchcres_articles

Part of the Medicine and Health Sciences Commons

\section{Recommended Citation}

Fei, Yurong; Xiao, Liping; and Hurley, Marja M., "The Impaired Bone Anabolic Effect of PTH in the Absence of Endogenous FGF2 is Partially Due to Reduced ATF4 Expression" (2011). UCHC Articles - Research. 139.

https://opencommons.uconn.edu/uchcres_articles/139 


\title{
The impaired bone anabolic effect of PTH in the absence of endogenous FGF2 is partially due to reduced ATF4 expression
}

\author{
Yurong Fei, Liping Xiao, and Marja M. Hurley* \\ University of Connecticut Health Center, Farmington, CT, USA \\ Yurong Fei: yfei@student.uchc.edu; Liping Xiao: xiao@uchc.edu; Marja M. Hurley: hurley@nso1.uchc.edu
}

Abstract

Parathyroid hormone (PTH) is currently the only approved anabolic agent for osteoporosis pharmacotherapy in the USA. However, the molecular and cellular mechanisms underlying which intermittent PTH stimulates bone formation are not fully established.

Activating transcription factor 4 (ATF4) was recently identified to be a downstream target of PTH signaling in osteoblasts and FGF2 is able to rapidly increase ATF4 mRNA and protein expression in osteoblasts. Furthermore, ATF4 expression is markedly reduced in $\mathrm{Fgf2}^{-/-}$osteoblasts. In addition, FGF2 is required for the anabolic action of PTH on bone formation. Therefore, we hypothesize that the impaired anabolic effect of PTH in $\mathrm{Fgf2}^{--}$mice is partially due to reduced ATF4 expression. To test this hypothesis, we examined the ability of PTH to increase ATF4 expression in vitro and in vivo. In vitro data showed that PTH induced a significant increase in ATF4 mRNA expression as early as $15 \mathrm{~min}$ in $\mathrm{Fgf}^{+/+}$primary bone marrow stromal cells

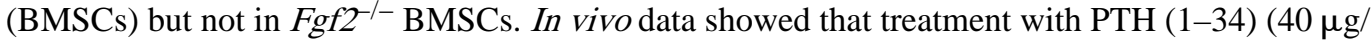
$\mathrm{kg} / \mathrm{d}$ ) treatment for 2 weeks in 21-23 months female mice increased lumbar vertebrae bone mineral density in $\mathrm{Fgf}^{+/+}\left(13.8 \%\right.$ increase). In contrast there was a $2.1 \%$ decrease in $\mathrm{Fgf2}{ }^{-/-}$ mice. Interestingly, basal ATF4 mRNA expression in tibiae was significantly lower in $\mathrm{Fgf2}^{-1-}$ mice (46\% decrease) compared to $F g f 2^{+/+}$mice. PTH treatment increased ATF4 mRNA by $97 \%$ $(\mathrm{p}<0.05)$ in $\mathrm{Fgf}^{+/+}$compared to $8 \%(\mathrm{p}=0.57)$ in $\mathrm{Fgf2}^{-/-}$mice. Immunohistochemistry of vertebrae showed less ATF4 staining in $\mathrm{Fgf}^{-/-}$tissue, and treatment with PTH increased ATF4 staining in $\mathrm{Fgf}^{2^{+/+}}$but the increase was attenuated in $\mathrm{Fgf2}^{-/-}$tissue.

In summary, reduced ATF4 expression may result in decreased osteoblast differentiation, and possibly contribute to the impaired stimulation of PTH on bone formation in $\mathrm{Fgf}^{-/-}$mice.

\section{Keywords}

FGF2; PTH; ATF4; Osteoblasts; Osteoporosis

\section{Introduction}

Osteoporotic fracture is estimated to occur every $3 \mathrm{~s}$ worldwide [1]. Osteoporosis is a disease characterized by low bone mass and deterioration in the microarchitecture of bone tissue [2]. Currently, the only available anabolic agent in clinical use for the treatment of osteoporosis in USA is PTH. Intermittent administration of PTH peptide 1-34 or full-length protein PTH 1-84 increases bone formation in osteoporotic patients [3]. However, the

\footnotetext{
(C) 2011 Elsevier Inc. All rights reserved.

* Corresponding author. Address: Department of Medicine, University of Connecticut Health Center, 263 Farmington Ave., Farmington, CT 06030, USA. Fax: +1 18606791875.
} 
molecular and cellular mechanisms underlying which intermittent PTH stimulates bone formation are not fully established.

Our previous studies demonstrate that anabolic action of PTH on bone formation requires FGF2. PTH increases FGF2 and FGF receptor mRNA expression in cultured osteoblasts [4]. Furthermore, PTH treatment increases serum FGF2 and increases bone formation markers in osteoporotic patients [5]. However disruption of FGF2 gene in mice results in dramatic reduction in bone formation [6], suggesting the importance of endogenous FGF2 in bone formation. Moreover, in $\mathrm{Fgf}^{---}$mice, PTH has impaired ability to stimulate bone formation [7]. These studies indicate that FGF2 is required for PTH to stimulate bone formation.

Bone formation is mediated by osteoblasts. Osteoblast differentiation from bone marrow stromal cell is governed by transcription factors, including Runx2 and ATF4 [8]. ATF4 is critical for osteoblast terminal differentiation and for maintaining mature osteoblast function including synthesizing collagen and osteocalcin [9]. Osteocalcin is one transcriptional target of ATF4 in osteoblasts [9]. The requirement of ATF4 in osteoblast differentiation is revealed by ATF4 knock out mice. Atf $4^{-/}$mice display a dramatic decrease in osteocalcin expression together with severe reduction of bone mass and bone formation [9]. In contrast, ATF4 overexpression increases osteocalcin expression in osteoblasts [10]. These studies suggest that ATF4 is a transcriptional activator required for osteoblast terminal differentiation and osteocalcin expression.

ATF4 has recently been identified as a novel downstream target gene of PTH signaling in osteoblasts [11,12]. PTH induces ATF4 mRNA/protein expression and increases ATF4 transcriptional activity in MC3T3-E4 cells and in BMSCs [11]. But PTH could not induce osteocalcin expression in ATF4 knockdown MC3T3-E4 cells or in Atf4 ${ }^{-1-}$ BMSCs [11]. Moreover, the anabolic actions of PTH in bone was severely impaired in $\mathrm{Atf4}^{--}$mice [12]. These results suggest that bone formation stimulated by PTH is in part mediated by ATF4.

We recently reported that ATF4 expression is regulated by FGF2 in osteoblasts. FGF2 is able to rapidly increase ATF4 mRNA and protein expression in osteoblasts [13].

Furthermore, ATF4 expression is markedly reduced in $\mathrm{Fgf2}^{-/-}$osteoblasts [13]. Therefore, we hypothesize that the impaired anabolic effect of PTH in $\mathrm{Fgf}^{-/-}$mice is partially due to reduced ATF4 expression. To test this hypothesis, we examined the ability of PTH to increase ATF4 expression both in vitro and in vivo.

In this study, we provide evidence that PTH is not able to induce ATF4 mRNA expression in the absence of FGF2 in BMSCs in vitro and PTH in vivo treatment increased ATF4 mRNA expression in tibia and ATF4 protein expression in vertebrae tissue is attenuated in FGF2 knockout mice. These results suggest that the impaired bone response to PTH in $\mathrm{Fgf2}^{-/-}$mice is in part due to reduced ATF4 expression.

\section{Materials and methods}

\subsection{Animals}

Fgf2 knockout mice were previously developed on a black swiss 129Sv background [14]. Mice used in this study were on a new mix genetic background of black swiss 129Sv/FVB/ $\mathrm{N}$. Genotyping of mice was performed using primers as previously described [14,15]. Mice were bred and housed in the transgenic facility in the center for laboratory animal care at the University of Connecticut Health Center. Mice were sacrificed by $\mathrm{CO}_{2}$ narcosis and cervical dislocation. The Animal Care and Use Committee of the University of Connecticut Health Center approved all animal protocols. 
Littermates of male or female mice were utilized as indicated in the figure legends. For PTH injection experiments, two independent studies were done using 17-19 month old mice $(\mathrm{n}=$ $3-4$ /group) or 21-23 month-old female mice ( $\mathrm{n}=2-3$ /group) that were weighed and injected subcutaneously with vehicle $(0.001 \mathrm{M} \mathrm{HCl}$ in PBS with $1 \mathrm{mg} / \mathrm{ml} \mathrm{BSA})(\mathrm{n}=2-3)$ or $40 \mu \mathrm{g} /$ kg human PTH (1-34) (Bachem, Torrence, CA, USA) 5×/week for 2 weeks. Two days after the last injection of $\mathrm{PTH}$, in vivo vertebral BMD and BMC were determined, and then the third vertebrae were placed in $10 \%$ phosphate-buffered formalin for $48 \mathrm{~h}$ and subsequently stored in $70 \%$ ethanol before immunohistochemistry staining. Left tibia were dissected and cut off the epiphyses for RNA extraction.

\subsection{Bone mineral density (BMD) and bone mineral content (BMC)}

In vivo vertebral $\mathrm{BMD}$ and $\mathrm{BMC}$ were determined using dual energy $\mathrm{X}$-ray absorptiometry (DXA; PIXimus mouse 11 densitometer, GE Medical System, Madison WI).

\subsection{RNA isolation and quantitative real-time PCR analysis}

Total RNA was extracted from cells with TRIzol reagent (Invitrogen, CA, USA) according to the manufacture's protocol. RNA $(3 \mu \mathrm{g})$ was reverse transcribed using a commercial kit (Clontech, CA, USA). Quantitative real-time PCR (qPCR) was carried out using the QuantiTect ${ }^{\mathrm{TM}}$ SYBR Green PCR kit using a MyiQ ${ }^{\mathrm{TM}}$ instrument (Bio-Rad, Laboratories Inc. Hercules, CA, USA). Glyceraldehyde 3-phosphate dehydrogenase (GAPDH) was used as an internal reference for each sample. Relative mRNA expression was calculated using a formula reported previously [16]. The mouse specific primers are as follows: Gapdh, $5^{\prime}$ CAG TGC CAG CCTCGT CCC GTA GA-3' (forward), 5' -CTG CAA ATG GCA GCC CTGGTG AC-3' (reverse); osteocalcin, $5^{\prime}$-GAG GGC AAT AAG GTA GTG AAC AGA-3' (forward), 5' -AAG CCA TAC TGG TTT GAT AGC TCG-3' (reverse); Type I collagen (col1a1), 5' -GGT CCT CGT GGT GCT GCT-3' (forward), 5' -ACC TTT GCC CCC TTC TTT G-3' (reverse); Atf4, 5' -GAG CTT CCT GAA CAG CGA AGT G-3' (forward), $5^{\prime}$-TGG CCA CCT CCA GAT AGT CAT C-3' (reverse).

\subsection{Immunohistochemistry staining}

For histological analysis, vertebrae samples incubated in $70 \%$ ethanol were decalcified in $20 \%$ EDTA in PBS for 7 days at room temperature and paraffin blocks were prepared by standard procedures. Samples were then cut into $5 \mu \mathrm{m}$ sections. Sections were deparaffinized and stained with ATF4 antibody (1:50, Santa Cruz, CA, USA) using ABC staining system (Santa Cruz, CA, USA) following the product protocol.

\subsection{Bone marrow stromal cell cultures}

For PTH in vitro treatment experiments, BMSC-isolation was described previously [13]. Briefly, femurs and tibiae from $\mathrm{Fgf2}{ }^{+/+}$and $\mathrm{Fgf2} 2^{-/-}$mice were dissected and both epiphyses were removed, then bones were centrifuged briefly in eppendorf tubes to release bone marrow cells. The marrow cells were plated at $10 \times 10^{6}$ cells/well in six-well plates in a MEM (Gibco-BRL, Grand Island, New York, USA) containing 10\% fetal bovine serum (FBS), penicillin (100 U/ml) and streptomycin $(50 \mu \mathrm{g} / \mathrm{ml})$. To study whether exogenous PTH promoted ATF4 expression, the cells had half medium changed on day 3 and the entire medium changed on day 6 with FBS-free a-MEM, with serum deprivation $14 \mathrm{~h}$ before adding exogenous $1 \mathrm{nM}$ PTH or vehicle for $15 \mathrm{~min}, 30 \mathrm{~min}, 1 \mathrm{~h}, 3 \mathrm{~h}$, or $6 \mathrm{~h}$. Then cells were harvest for RNA extraction and gene analysis as describe above. 


\subsection{Statistical analysis}

Results were expressed as the means \pm SEM. Differences between wild type vs knockout or vehicle vs treatment were analyzed using a student's $t$-test, and differences were considered significant at $p<0.05$.

\section{Results}

\subsection{PTH is not able to increase ATF4 mRNA expression in the absence of endogenous FGF2 in BMSCs in vitro}

The ability of PTH to increase ATF4 expression was first examined in BMSCs. As shown in Fig. 1, ATF4 mRNA expression is significantly reduced in $\mathrm{Fgf2}^{-/-}$BMSCs compared to $\mathrm{Fgf}^{+/+}$BMSCs at basal level. PTH treatment significantly increased ATF4 mRNA expression in $\mathrm{Fgf2}^{+/+}$BMSCs as early as $15 \mathrm{~min}$. However, PTH was not able to increase ATF4 mRNA expression in $\mathrm{Fgf2}^{-/-}$BMSCs at all the time points examined.

\subsection{Anabolic action of PTH on bone formation is impaired in $\mathrm{Fgf2}^{-/-}$mice compared to wild type littermates}

We next assessed bone formation stimulated by PTH in 21-23 month-old female mice on a black swiss $129 \mathrm{~Sv} / \mathrm{FVB} / \mathrm{N}$ genetic background. We observed that PTH treatment increased lumbar vertebrae BMD in $\mathrm{Fgf2}^{+/+}$mice with a $13.8 \%$ increase. In contrast there was a $2.1 \%$ decrease in $\mathrm{Fgf}^{-/-}$mice (Fig. 2A). PTH treatment increased lumbar vertebrae BMC with a 19.8\% increase in $\mathrm{Fgf2}^{+/+}$mice but there was a 9.9\% decrease in $\mathrm{Fgf2}^{-/-}$mice (Fig. 2B).

We also examined genes that are important for osteoblasts differentiation using RNA extracted from tibiae after PTH in vivo administration. For Colla1 mRNA expression (Fig. 2C), PTH induced a $363 \%$ increase in $\mathrm{Fgf}^{+/+}$mice vs a $118 \%$ in $\mathrm{Fgf}^{-/-}$mice. As shown in (Fig. 2D), PTH induced a $221 \%$ increase in osteocalcin expression in $\mathrm{Fgf}^{+/+}$mice vs $173 \%$ in Fgf $2^{-1-}$ mice. Also (Fig. 2E), PTH injection induced a 51\% increase in Runx2 mRNA expression in $\mathrm{Fgf2}^{+/+}$mice vs $10 \%$ in $\mathrm{Fgf2}^{-/-}$mice. These data showed that increased gene expression of osteocalcin, Col1a1 and Runx2 stimulated by PTH injection were attenuated in $\mathrm{Fgf2}^{-/-}$mice compared to $\mathrm{Fgf}^{+/+}$mice. Impaired bone anabolic response to PTH was also seen in 17-19 month-old female mice with $16.9 \%$ increase in vertebrae BMD in $\mathrm{Fgf}^{+/+}$mice vs $6.2 \%$ increase in $\mathrm{Fgf2}^{-/-}$mice.

\subsection{Increased ATF4 mRNA and protein expression by PTH injection is attenuated in $\mathrm{Fgf2}^{-/-}$mice compared to wild type littermates}

Since the anabolic action of PTH on bone formation requires ATF4, we investigated the ability of PTH to increase ATF4 expression in vivo in 21-23 month old female $\mathrm{Fgf2}^{-/-}$mice compared to wild type littermates. Interestingly, ATF4 mRNA expression in tibiae was significantly lower in $\mathrm{Fgf2}^{-/-}$mice (a $46 \%$ decrease) compared to $\mathrm{Fgf2}^{+/+}$mice. PTH treatment increased ATF4 mRNA expression by $97 \%(\mathrm{p}<0.05)$ in $\mathrm{Fgf2}^{+/+}$compared to $8 \%$ ( $\mathrm{p}=0.57)$ in $\mathrm{Fgf2}^{-/-}$mice, as shown in Fig. 3A.

We further examined ATF4 protein in vivo expression by immunohistochemistry on lumbar vertebrae after PTH injection. As shown in Fig. 3B, there was less ATF4 staining in $\mathrm{Fgf2}^{-/-}$ vertebrae tissue, particularly in osteoblast cells lining the bone surface (arrow) compared to $\mathrm{Fgf2}^{+/+}$tissue, and treatment with PTH increased ATF4 staining in $\mathrm{Fgf}^{+/+}$but the increase was attenuated in $\mathrm{Fgf2}^{-/-}$tissue. 


\section{Discussion}

In the present study, we found that PTH did not increase ATF4 mRNA expression in the absence of endogenous FGF2 in BMSCs in vitro. Furthermore, increased ATF4 mRNA in tibia and ATF4 protein expression in vertebrae tissue by PTH injection is attenuated in Fgf $2^{-/-}$mice compared to wild type littermates. Attenuated ATF4 expression may contribute to the impaired anabolic actions of PTH on bone formation in $\mathrm{Fgf2}^{-\mathrm{-}}$ mice.

The impaired bone anabolic response of PTH in $\mathrm{Fgf2}^{-/-}$mice on a new genetic background of black swiss/129Sv/FVB/N is similar to the results reported in mice on a black swiss/ 129Sv genetic background [7]. One also needs to note that the dose of PTH treatment in the current study $40 \mu \mathrm{g} / \mathrm{kg}$ for $5 \times /$ week for 2 weeks was much lower that the dose used in our previous studies $(80 \mu \mathrm{g} / \mathrm{kg}$ once daily for 4 weeks or $160 \mu \mathrm{g} / \mathrm{kg}$ for 10 days). These data further support that the anabolic bone response to PTH requires FGF2.

We previously reported that PTH treatment increased serum FGF2 and increased bone formation markers in osteoporotic patients [5]. In cultured osteoblasts, PTH increased FGF2 and FGF receptor mRNA expression [4]. The increased bone formation stimulated by PTH may be due to increased FGF2, since the ability of PTH to stimulate bone formation is impaired in the absence of endogenous FGF2 [7]. FGF2 positively regulated osteoblast differentiation and bone formation. Disruption of the Fgf2 gene resulted in significantly decreased bone mass and bone formation as revealed by histomorphometry and micro-CT $[15,17]$. Decreased osteoblast differentiation might be due to reduced expression of transcription factors, since we found that reduced ATF4 expression in osteoblasts was associated with reduced bone formation in $\mathrm{Fgf}^{-/-}$mice [13]. Furthermore, exogenous FGF2 was able to induce ATF4 expression [13]. These results suggest that ATF4 may be a down stream target of FGF2 signaling in osteoblasts (Fig. 4).

ATF4 is a transcriptional activator for osteoblasts differentiation. Expression of the latest marker of differentiated osteoblasts osteocalcin and synthesis of the major constituent of the bone matrix type I collagen require ATF4 [9]. Atf4 deficient mice display reduced type I collagen synthesis, decreased osteocalcin expression and low bone mass compared to wild type [9]. Therefore, ATF4 is an essential regulator of osteoblast biology. Recent studies demonstrated that ATF4 is critical in the anabolic actions of PTH in bone [11,12]. PTH increased osteocalcin expression in $\mathrm{Atf4}^{-/}$BMSCs was attenuated [12] and PTH stimulated bone formation in Atf4 deficient mice was severely impaired [13]. Thus, PTH signaling in bone is in part mediated by ATF4.

To our knowledge, this is the first report that the impaired bone anabolic response of PTH in the absence of FGF2 is associated with attenuated ATF4 expression. The attenuated ATF4 expression may contribute to reduced osteoblast differentiation with decreased type I collagen and osteocalcin expression and reduced bone formation (Fig. 4). Future studies will address whether ATF4 over-expression is able to rescue the impaired anabolic actions of PTH on bone formation in Fgf 2 knockout mice.

\section{Acknowledgments}

This project was supported by NIH grant AG021189 to Marja M. Hurley.

\section{References}

1. Johnell O, Kanis JA. An estimate of the worldwide prevalence and disability associated with osteoporotic fractures. Osteoporos Int. 2006; 17:1726-1733. [PubMed: 16983459] 
2. Holroyd C, Cooper C, Dennison E. Epidemiology of osteoporosis. Best Pract Res Clin Endocrinol Metab. 2008; 22:671-685. [PubMed: 19028351]

3. Hodsman AB, Bauer DC, Dempster DW, et al. Parathyroid hormone and teriparatide for the treatment of osteoporosis: a review of the evidence and suggested guidelines for its use. Endocr Rev. 2005; 26:688-703. [PubMed: 15769903]

4. Hurley MM, Tetradis S, Huang YF, et al. Parathyroid hormone regulates the expression of fibroblast growth factor- 2 mRNA and fibroblast growth factor receptor mRNA in osteoblastic cells. J Bone Miner Res. 1999; 14:776-783. [PubMed: 10320526]

5. Hurley MM, Yao W, Lane NE. Changes in serum fibroblast growth factor 2 in patients with glucocorticoid-induced osteoporosis treated with human parathyroid hormone (1-34). Osteoporosis Int. 2005; 16:2080-2084.

6. Naganawa T, Xiao L, Abogunde E, Sobue T, Kalajzic I, Sabbieti M, Agas D, Hurley MM. in vivo and in vitro comparison of the effects of FGF-2 null and haplo-insufficiency on bone formation in mice. Biochem Biophys Res Commun. 2006; 339:490-498. [PubMed: 16298332]

7. Hurley MM, Okada Y, Xiao L, Tanaka Y, Ito M, Okimoto N, Nakamura T, Rosen CJ, Doetschman T, Coffin JD. Impaired bone anabolic response to parathyroid hormone in $\mathrm{Fgf2}^{-/-}$and $\mathrm{Fgf2+} /-$ mice. Biochem Biophys Res Commun. 2006; 341:989-994. [PubMed: 16455048]

8. Karsenty G. Transcriptional control of skeletogenesis. Annu Rev Genom Hum G. 2008; 9:183-196.

9. Yang X, Matsuda K, Bialek P, et al. ATF4 is a substrate of RSK2 and an essential regulator of osteoblast biology: implication for Coffin-Lowry syndrome. Cell. 2004; 117:387-398. [PubMed: 15109498]

10. Xiao G, Jiang D, Ge C, et al. Cooperative interactions between activating transcription factor 4 and Runx2/Cbfa1 stimulate osteoblast-specific osteocalcin gene expression. J Biol Chem. 2005; 280:30689-30696. [PubMed: 16000305]

11. Yu S, Franceschi RT, Luo M, et al. Parathyroid hormone increases activating transcription factor 4 expression and activity in osteoblasts: requirement for osteocalcin gene expression. Endocrinology. 2008; 149:1960-1968. [PubMed: 18187540]

12. Yu S, Franceschi RT, Luo M, et al. Critical role of activating transcription factor 4 in the anabolic actions of parathyroid hormone in bone. PLoS ONE. 2009; 4:e7583. [PubMed: 19851510]

13. Fei Y, Xiao L, Hurley MM. Fibroblast growth factor 2 positively regulates expression of activating transcription factor 4 in osteoblasts. Biochem Biophys Res Commun. 2010; 391:335-339. [PubMed: 19913500]

14. Zhou M, Sutliff RL, Paul RJ, et al. Fibroblast growth factor 2 control of vascular tone. Nat Med. 1998; 4:201-207. [PubMed: 9461194]

15. Montero A, Okada Y, Tomita M, Ito M, Tsurukami H, Nakamura T, Doetschman T, Coffin JD, Hurley MM. Disruption of the fibroblast growth factor-2 gene results in decreased bone mass and bone formation. J Clin Invest. 2000; 105:1085-1093. [PubMed: 10772653]

16. Pfaffl M. A new mathematical model for relative quantification in real-time RT-PCR. Nucleic Acids Res. 2001; 29:e45. [PubMed: 11328886]

17. Xiao L, Sobue T, Esliger A, Kronenberg MS, Coffin JD, Doetschman T, Hurley MM. Disruption of the Fgf2 gene activates the adipogenic and suppresses the osteogenic program in mesenchymal marrow stromal stem cells. Bone. 2010; 47:360-370. [PubMed: 20510392] 


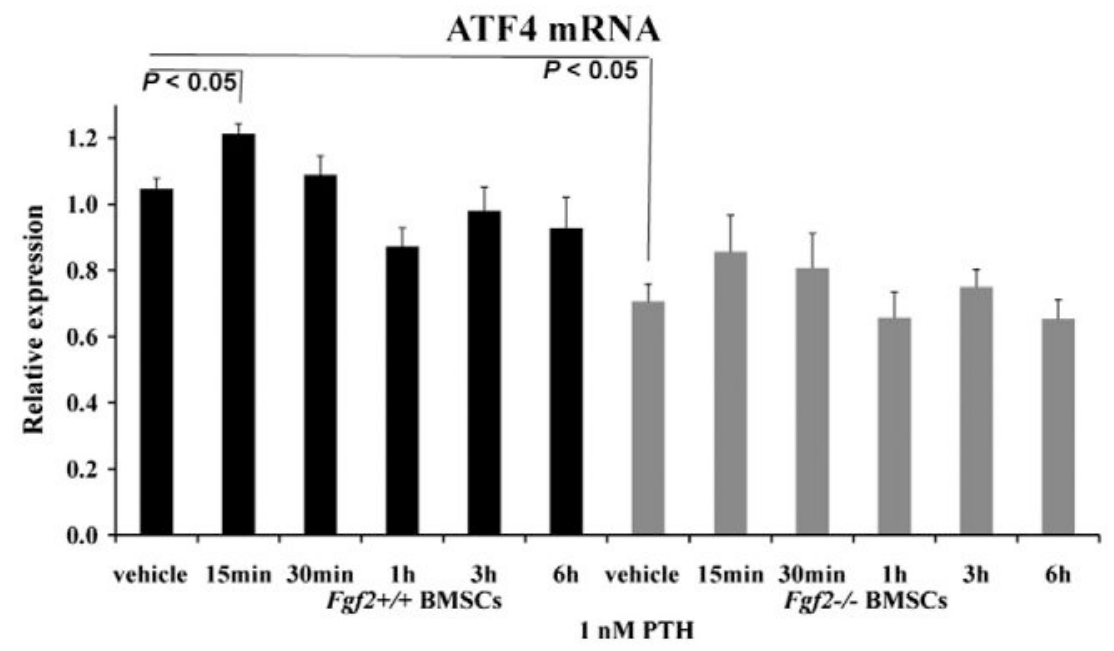

Fig. 1.

PTH is not able to increase ATF4 mRNA expression in the absence of endogenous FGF2 in BMSCs in vitro. ATF4 mRNA expression is significantly reduced in $\mathrm{Fgf2}^{-/}$BMSCs compared to $\mathrm{Fgf2}^{+/+}$BMSCs at basal level. PTH treatment significantly increased ATF4 mRNA expression in $\mathrm{Fgf2}^{+/+}$BMSCs as early as $15 \mathrm{~min}$. However, PTH does not increase ATF4 mRNA expression in $\mathrm{Fgf2}^{-/-}$BMSCs at the time points examined. Freshly isolated BMSCs from 3 months old male mice were cultured in a MEM with 10\% FBS for 6 days, serum deprived $14 \mathrm{~h}$, then treated with $1 \mathrm{nM}$ PTH or vehicle at the indicated times, followed by total RNA extraction for gene analysis. Data are mean \pm SEM for two to three independent experiments. 

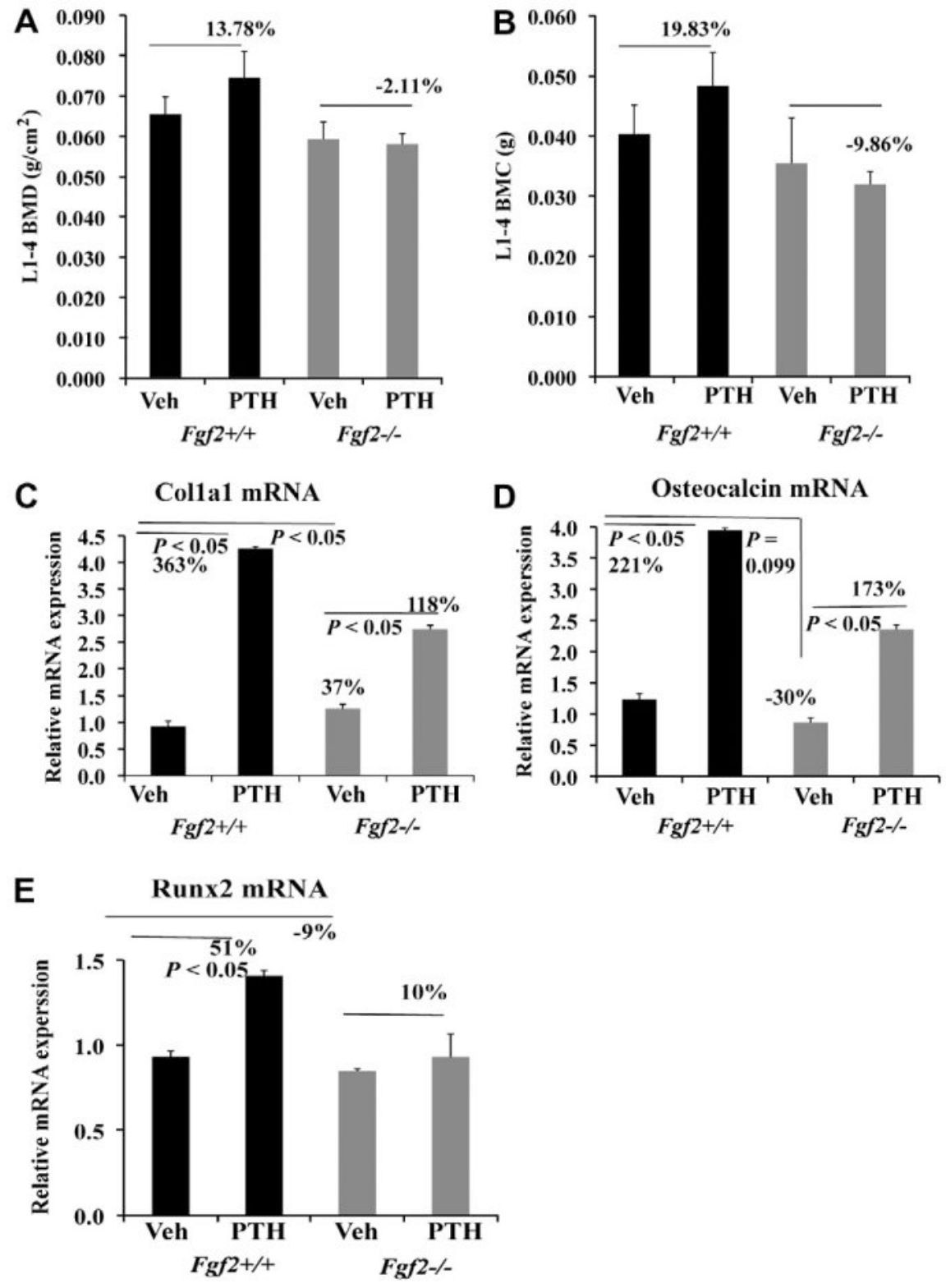

Fig. 2.

Anabolic action of PTH on bone formation is impaired in $\mathrm{Fgf2}^{-/-}$mice compared to wild type littermates on a new mix genetic background of black swiss $129 \mathrm{~Sv} / \mathrm{FVB} / \mathrm{N}$. Increased vertebral BMD (A) and BMC (B) by PTH injection is attenuated in $F g f 2^{-1-}$ mice. PTH stimulation of genes important in osteoblast differentiation; type I collagen Colla1 (C), osteocalcin (D) and Runx2 (E) by PTH is attenuated in tibia of $F g f 2^{-1-}$ mice. $21-23$ ms old female mice were injected subcutaneously $5 \times$ /week for two weeks with either vehicle or PTH (40 lg/kg BW) as described in materials and methods. Two days after the last injection, BMD and BMC were determined and total RNA was extracted from whole tibia excluding epiphyses for gene analysis by qPCR. 
A
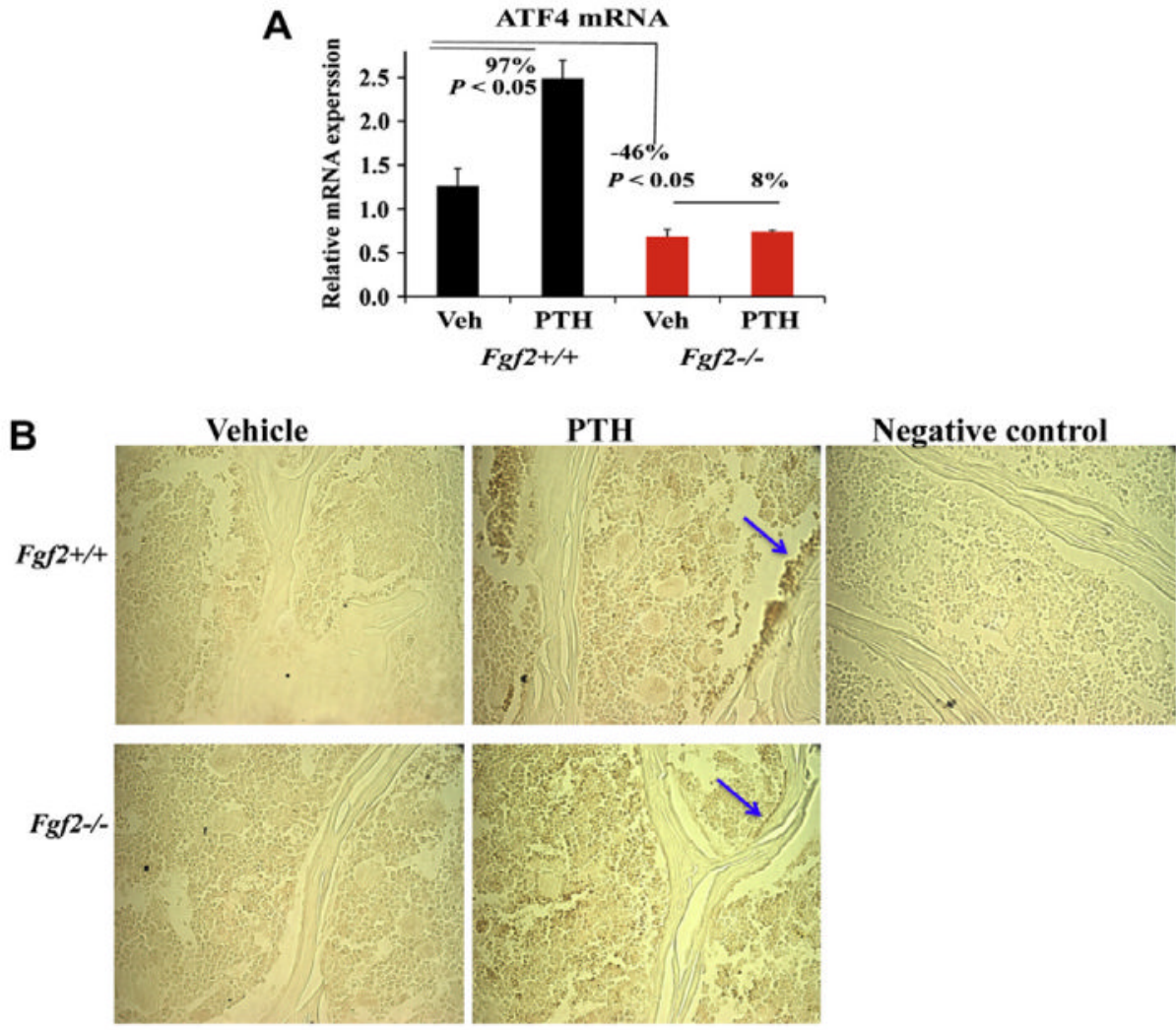

Fig. 3.

Increased ATF4 mRNA and protein expression in vertebrae tissue by PTH injection is attenuated in $\mathrm{Fgf}^{-/-}$mice compared to wild type littermates. 21-23 months old female mice were injected subcutaneously $5 \times /$ week for two weeks with either vehicle or PTH $(40 \mu \mathrm{g} / \mathrm{kg}$ $\mathrm{BW})$ as described in materials and methods. Two days after the last injection, total RNA was extracted from whole left tibia excluded epiphyses for ATF4 analysis by qPCR (A). (B) Immunohistochemistry for ATF4 protein expression in vertebrae tissue and in osteoblast cells lining on bone surface (arrow) (maganification, 400x). Representative image of vertebrae from each treatment group and genotype are shown. 


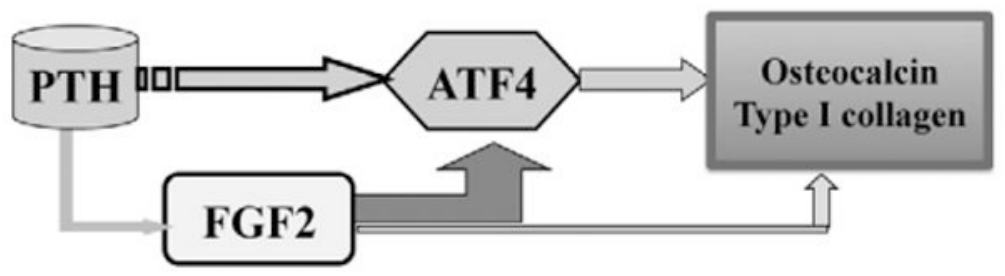

Fig. 4.

Schematic model - The impaired bone anabolic effect of PTH in Fgf2 knockout mice is associated with reduced ATF4 expression. Anabolic action of PTH on bone formation requires FGF2 and FGF2 increases ATF4 expression and transcriptional activity in osteoblasts. Moreover, ATF4 is a downstream target of PTH signaling in osteoblasts and ATF4 is critical for osteoblasts differentiation. Therefore, attenuated ATF4 expression in $\mathrm{Fgf2}^{-/-}$mice by PTH injection may contribute to reduced osteoblasts differentiation characterized by decreased osteocalcin and type I collagen expression, which results in impaired stimulation of bone formation by PTH. 